\title{
BRAF mutation in hairy cell leukemia
}

\author{
Ahmad Ahmadzadeh, ${ }^{1}$ Saeid Shahrabi, ${ }^{2}$ Kaveh Jaseb, ${ }^{1}$ Fatemeh Norozi, ${ }^{1}$ \\ Mohammad Shahjahani, ${ }^{1}$ Tina Vosoughi, ${ }^{1}$ Saeideh Hajizamani, ${ }^{1}$ Najmaldin Saki ${ }^{1}$ \\ ${ }^{1}$ Health Research Institute, Research Center of Thalassemia and Hemoglobinopathy, Ahvaz \\ Jundishapur University of Medical Sciences, Ahvaz; ${ }^{2}$ Department of Biochemistry and Hematology, \\ Semnan University of Medical Sciences, Semnan, Iran
}

\begin{abstract}
BRAF is a serine/threonine kinase with a regulatory role in the mitogen-activated protein kinase (MAPK) signaling pathway. A mutation in the $R A F$ gene, especially in BRAF protein, leads to an increased stimulation of this cascade, causing uncontrolled cell division and development of malignancy. Several mutations have been observed in the gene coding for this protein in a variety of human malignancies, including hairy cell leukemia (HCL). BRAF V600E is the most common mutation reported in exon15 of BRAF, which is observed in almost all cases of classic HCL, but it is negative in other B-cell malignancies, including the HCL variant. Therefore it can be used as a marker to differentiate between these B-cell disorders. We also discuss the interaction between miRNAs and signaling pathways, including MAPK, in HCL. When this mutation is present, the use of BRAF protein inhibitors may represent an effective treatment. In this review we have evaluated the role of the mutation of the $B R A F$ gene in the pathogenesis and progression of HCL.
\end{abstract}

Correspondence: Najmaldin Saki, Health Research Institute, Research Center of Thalassemia and Hemoglobinopathy, Ahvaz Jundishapur University of Medical Sciences, Ahvaz, Iran.

Tel. +98.6113738317 - Fax: +98.6113738330 .

E-mail: najmaldinsaki@gmail.com

Key words: BRAF mutation, MAPK signaling pathway, hairy cell leukemia, miRNA.

Contributions: NS, AA and SH conceived and revised the manuscript. FN, MSh, SSh, TV and KJ wrote the manuscript. All authors approved the final version of the manuscript.

Conflict of interests: the authors declare no conflict of interests.

Acknowledgments: we wish to thank all our colleagues in Shafa Hospital and Allied Health Sciences School, Ahvaz Jundishapur University of Medical Sciences.

Received for publication: 4 May 2014.

Revision received: 6 July 2014.

Accepted for publication: 8 July 2014.

This work is licensed under a Creative Commons Attribution NonCommercial 3.0 License (CC BY-NC 3.0).

(C) Copyright A. Ahmadzadeh et al., 2014

Licensee PAGEPress, Italy

Oncology Reviews 2014; 8:253

doi:10.4081/oncol.2014.253

\section{Introduction}

The mitogen-activated protein kinase (MAPK) is a pathway that regulates proliferation, differentiation, development, survival and apoptosis in response to growth factors, cytokines and hormones in mammalian cells. ${ }^{1,2}$ Several protein kinases, including the BRAF protein, are involved in this pathway. BRAF is a serine/threonine kinase from the RAF kinase family encoded by the $B R A F$ gene on chromosome 7 at $7 \mathrm{q} 34$ position, which has a regulatory role in the activation of MAPK/extracellular-signal-regulated kinase (ERK) signaling pathway (Figure 1A). ${ }^{3,4}$ ERK/MAP kinase pathway is activated by a variety of receptors, including receptor tyrosine kinases and G-proteins, which, when stimulated, lead to the activation of the small G-protein of Ras. ${ }^{5}$ Ras is an upstream activator of the Raf family of protein kinases (ARaf/BRaf/CRaf). ${ }^{6}$ The three Raf kinases can all activate MEK1/2, which in turn activates ERK1/2, resulting in the phosphorylation of target proteins, including fos in, the nucleus and the transcription factors activator protein 1 (AP-1) and nuclear factor of activated T-cells (NFAT), which eventually lead to cell proliferation, differentiation and survival. ${ }^{1,7}$ BRAF mutations directly cause activation of MEK and signal a transfer to ERK, leading to the activation of this pathway. ${ }^{8}$ Excessive activation of this pathway leads to an uncontrolled cell cycle and plays an oncogenic role in human cancers. ${ }^{9}$ Approximately 43 mutations have been identified in exons 11 and 15 of the BRAF gene, which are associated with a variety of human malignancies, the majority of which cause the activation of the kinase domain of this protein. ${ }^{10}$ The most common BRAF mutation is V600E, which is caused by the substitution of adenine (A) for thymine (T) at position 1799 on exon 15 and results in the change of amino acid 600 from valine to glutamate in the protein sequence, followed by a constant activity of the downstream kinases, independently of the extracellular signals and increased cell proliferation (Figure 1B). ${ }^{11,12}$ Hairy cell leukemia (HCL) has this type of pattern, and this mutation has been found in almost all classic cases of this malignancy.

HCL is a rare lymphoproliferative disorder of B-cells, and accounts for approximately $2 \%$ of leukemic diseases. ${ }^{13}$ It is characterized by a progressive pancytopenia, splenomegaly without lymphadenopathy, presence of B cells with abnormal cytoplasm and a hairy look with infiltration in bone marrow, liver and spleen. ${ }^{14,15}$ According to the 2008 World Health Organization (WHO) classification, diagnosis and differentiation of HCL from similar diseases, including splenic marginal zone lymphoma (SMZL), splenic lymphoma/leukemia unclassifiable, such as HCL variant (HCL-v), are based on morphological and immunophenotypic markers. ${ }^{14}$ Malignant cells co-express CD20, CD22, CD11c, CD25, CD103, tartrate-resistant acid phosphatase and Anexin A1. ${ }^{16,17}$ Classic HCL responds to purine nucleoside analogs, while HCL-v cases are resistant and are more aggressive compared with the classic variant. ${ }^{18}$ Therefore distinguishing classic HCL from 
other B-cell lymphomas, including HCL-v and SMZL, is critical, since treatment with purine nucleoside analogues is only effective in HCL. In this review, the association between HCL and BRAF mutation as well as miRNA will be discussed. We also show that targeting the BRAF oncogene with inhibitors provides a new therapeutic strategy, and BRAF mutation testing can be used for laboratory diagnosis of HCL.

\section{BRAF mutation and hairy cell leukemia}

Increased activity of MAPK pathway has been reported in a small number of patients with HCL, and plays a role in the growth and survival of HCL cells. ${ }^{19}$ The presence of BRAF V600E mutation leads to increased phosphorylation of MEK and ERK, followed by increased phosphorylation of AP-1 and NFAT transcription factors. ${ }^{1,7}$ AP-1 activation has been associated with the expression of HCL marker CD11c. ${ }^{20}$ Tiacci et al. found the BRAF V600E mutation in exon 15 of BRAF gene in $100 \%$ of classic HCL cases $(n=48)$ using Sanger sequencing, but not in other B-cell lymphomas/leukemias. ${ }^{21}$ Blombery et al. in their study confirmed the detection of BRAF V600E mutation in all classic HCL patients. In addition, they observed BRAF V600E mutation in patients with morphologically classic HCL with immunophenotypic variations $\left(\mathrm{CD} 25^{-}, \mathrm{CD} 10^{+}, \mathrm{CD} 123^{-}\right) .{ }^{22}$ In addition, in a study by Arcaini et al., BRAF V600E mutation was detected in all 62 HCL patients and only in 2 patients with other hematological malignancies. ${ }^{23}$ This mutation is not detected in HCL-v and IGHV4-34 variants of HCL. ${ }^{24}$ These findings confirmed that BRAF mutation V600E is a molecular hallmark of HCL. Two novel mutations in exon 11 of $B R A F$ gene were detected in BRAF V600E-negative HCLs. ${ }^{25}$ It has been suggested to screen exon 11 in V600E-negative HCL cases. BRAF mutation was present in hematopoietic stem cells in patients with HCL. ${ }^{26}$

\section{Hairy cell leukemia and miRNA}

MicroRNAs are small 19-22 nucleotide RNA molecules involved in the regulation of processes such as proliferation, apoptosis and suppression of target gene expression. ${ }^{27,28}$ MiRNA expression is altered in cancer. ${ }^{29}$ BRAF mutation may lead to a changing expression of several miRNAs with regulatory roles for several oncogenes, including BRAF. In recent years, the relationship between miRNA and a mutation in the $B R A F$ gene with a number of human cancers including papillary thyroid carcinoma (PTC) has been recognized. It was demonstrated that the expression of miR-221, miR-146b and miR-222 was higher in patients with BRAF mutation, and the invasiveness and the metastasis were more severe among them..$^{30}$ In 2012, Zhou et al. showed that the expression level of micRNA-221 was higher in PTC patients with a mutation in $B R A F$ gene, which can be involved in the extra-thyroid invasion of the tumor, tumor size and disease progression rate. ${ }^{31}$ The contribution of miRNAs to HCL has been analyzed by Kitagawa et al. in 2012. They identified miRNA, -221/-222 family, $-22,-24,27 \mathrm{a}$ and Let-7b in a higher level of expression in HCLs compared with normal and other malignant B-cells. ${ }^{32}$ They also suggested that miR-221/-222 overexpression in HCL may lead to a low expression of CDKN1B (p27/Kip1), which is a direct target of these miRNAs. ${ }^{33}$ On the basis of the association between BRAF mutation and miR-221/-222 in PTC, 30 a high level of this miRNA in HCL suggests that miR-221/-222 overexpression in HCL regulates MAPK signaling in HCL, and correlates with BRAF mutation. In chronic lymphocytic leukemia (CLL), cell proliferation is due to the overexpression of miR-22, which induces phosphatase and tensin analog down regulation and PI3-Akt pathway activation. ${ }^{34}$ In addition there is an upregulation in Cyclin D2 (CCND2) and MAPK1 genes. ${ }^{34} \mathrm{As}$ a consequence, we can suggest that miR-22 may modulate MAPK signaling in HCL and requires further investigation. MiR-24 activates

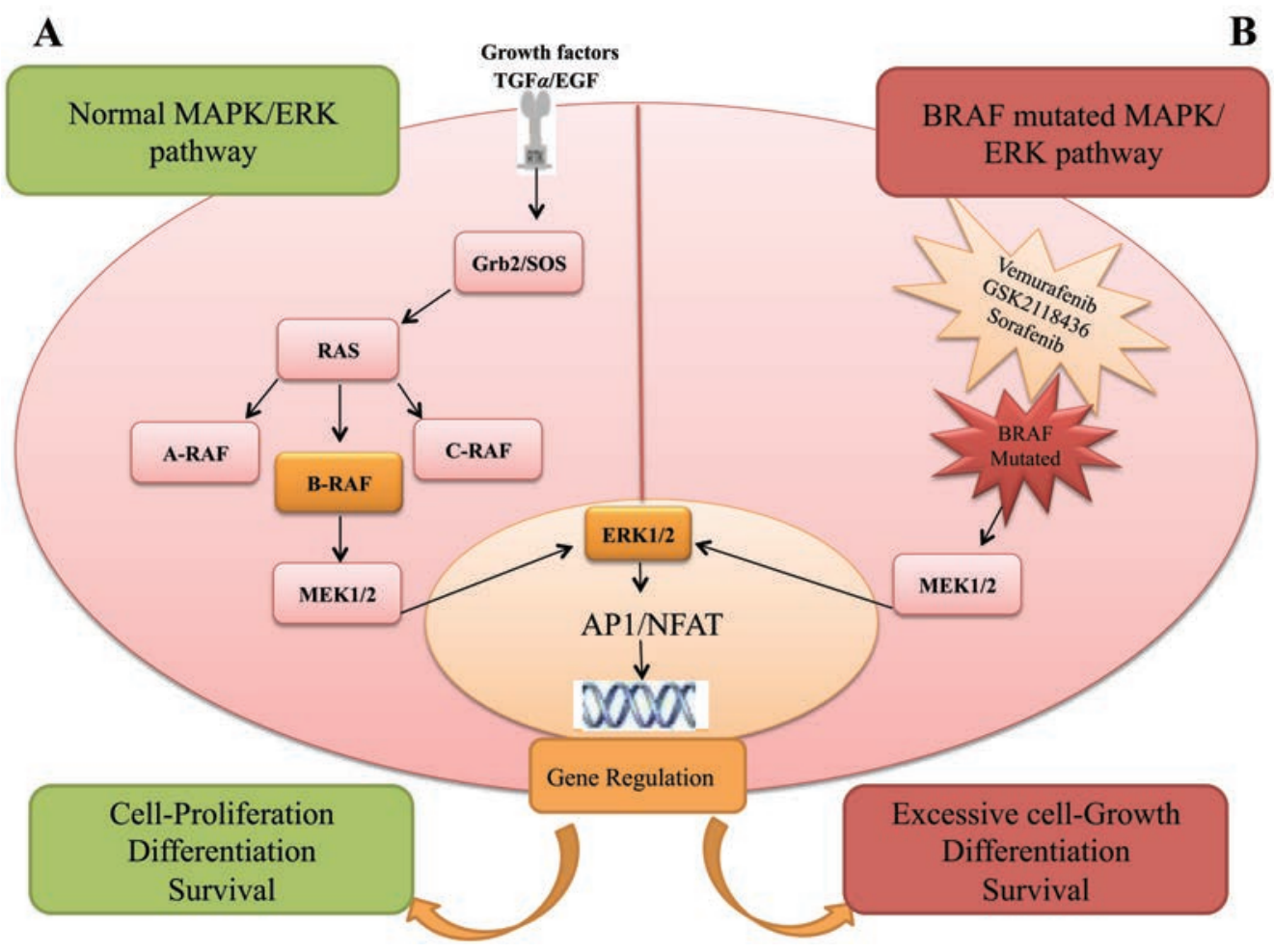

Figure 1. Role of BRAF in (A) normal and (B) mutated state in the activation of MAPK pathway. MAPK inhibitor drugs, such as vemurafenib, inhibit this protein by binding the mutated BRAF molecule, thus halting its activity and an excessive activation of the MAPK pathway. 
MAPK signaling by downregulating MAPK phosphatase 7 in acute myeloid leukemia. ${ }^{35}$ Therefore miR-24 is also related to MAPK signaling and can contribute to BRAF mutation in HCL. In the study of Moussay et al., the level of miR-363 and miR-708 was lower and higher in HCL and CLL, respectively. Also, there were no significant changes in miR-34a and miR-564 in HCL compared with normal subjects. ${ }^{36}$ MiRNA deregulation may be associated with prognosis and disease progression in HCL. The combined evaluation of the HCL-specific miRNA signature and its association with MAPK signaling may be useful to better understand the pathogenesis and management of HCL.

\section{Treatment and control of mutant BRAF effects}

Due to the importance of Ras and BRAF in the development of malignancies, Raf inhibitors have been generated especially against BRAF V600E. ${ }^{37}$ BRAF inhibition results in cell death by suppressing the Ras/Raf/MEK/ERK pathway in HCL. ${ }^{38}$ In in vitro studies, it was demonstrated that HCL cells undergo apoptosis with BRAF inhibitors. ${ }^{39}$ Vemurafenib, which inhibits thymidine kinase enzyme, has been recently approved for the treatment of patients with metastatic melanoma with BRAF V600E mutation after a randomized phase III trial. The overall survival and progression-free survival was improved in this group of patients. ${ }^{40}$ Dietrich et al. confirmed the mutant BRAF as a therapeutic target in HCL by using vemurafenib in patient with refractory HCL. ${ }^{41,42}$ Vemurafenib led to complete clinical remission by reducing the viability of CD-103+ ${ }^{+}$HCL cells. ${ }^{38}$ Other inhibitor compounds are also currently used in treatment of HCL (Table 1), ${ }^{43,44}$ which mainly bind the BRAF molecule and prevent MEK and ERK phosphorylation and cell division. The above-mentioned material validates the mutant BRAF as a therapeutic target in HCL.

\section{Discussion}

$B R A F$ is a commonly mutated gene in a variety of cancers. ${ }^{9}$ BRAF V600E mutation occurs in $40 \%$ to $70 \%$ of malignant melanomas, $45 \%$ of PTC and 5-15\% of colorectal cancers, $35 \%$ of ovarian tumors and $1-3 \%$ of cases of non-small cell lung carcinoma. ${ }^{45,46}$ The most common BRAF mutation is BRAF V600E, which results in the constant activity of downstream kinases, independently of extracellular signals and increased cell proliferation. ${ }^{11}$ In hematological malignancies, BRAF V600E has been defined as a genetic lesion in HCL. ${ }^{21}$ Until now, almost all cases of HCL (100\%) show this mutation. However, BRAF V600E mutation is not detected in other B-cell malignancies, including SMZL, HCL-v, CLL, mantle cell lymphoma and Waldenstrom macroglobulinemia. ${ }^{21,47}$ Therefore, BRAF V600E mutation may be a hallmark of HCL. Regulation

Table 1. Binding site of a number of inhibitor drugs of the MAPK pathway.

\begin{tabular}{lcc}
\hline Drugg (inhibitors) & Binding site & Ref \\
Vemurafenib or (PLX4032, PG7204, R05185436) & BRAF V600E & 43 \\
Sorafenib & RAF & 43 \\
\hline GSK2118436 & RAF & 43 \\
AZD6244 & MEK & 43 \\
\hline GSK1120212 & MEK & 43 \\
SB590885 & BRAF & 44 \\
\hline
\end{tabular}

of the MAPK pathway by a specific miRNA signature has been recently reported in HCL by Kitagawa et al. ${ }^{32}$ A deeper understanding of the relationship between miRNA and signaling pathways like MAPK may improve our knowledge of HCL pathogenesis. The function of miRNAs in HCL and their association with the MAPK pathway needs further investigation, as it may be used as a biomarker or regulator and enable clinical applications in HCL.

The use of inhibitor drugs that specifically bind to mutant BRAF molecules and halt the continuation of the pathway is effective in preventing disease progression. The clinical efficacy of BRAF kinase inhibitors has been reported in melanoma. ${ }^{48,49}$ BRAF inhibition discontinues the constant activation of the MAPK pathway, thus offering a new therapeutic opportunity in the treatment of BRAF V600E refractory HCL. ${ }^{36}$ Dietrich et al. confirmed the role of mutant BRAF as therapeutic target in HCL, using vemurafenib in patients with refractory HCL. 41,42

We can therefore conclude that BRAF V600E mutation, which has been found in almost all cases of classic HCL, can be used as a marker to differentiate this disease from similar diseases, such as HCL-v. However the evaluation of the BRAF mutation is not a routine clinical test for HCL, but molecular detection of BRAF V600E may be helpful in the diagnosis of HCL patients and in their follow up after treatment in order to monitor the disease and detect relapse. Further studies are required to establish the clinical benefit of this mutation in HCL.

\section{References}

1. Zhang W, Liu HT. MAPK signal pathways in the regulation of cell proliferation in mammalian cells. Cell Res 2002;12:9-18.

2. Wan PT, Garnett MJ, Roe SM, et al. Mechanism of activation of the RAF-ERK signaling pathway by oncogenic mutations of B-RAF. Cell 2004;116:855-67.

3. Akarca AU, Shende VH, Ramsay AD, et al. BRAF V600E mutation specific antibody, a sensitive diagnostic marker revealing minimal residual disease in hairy cell leukaemia. Br J Haematol 2013;162:848-51.

4. Burger JA. BRAF mutation: supporting diversity in HCL. Blood 2012;119:3193-4.

5. Gutkind JS. The pathways connecting G protein-coupled receptors to the nucleus through divergent mitogen-activated protein kinase cascades. J Biol Chem 1998;273:1839-42.

6. Cseh B, Doma E, Baccarini M. "RAF" neighborhood: protein-protein interaction in the Raf/Mek/Erk pathway. FEBS Lett 2014 [In press].

7. Flockhart R, Armstrong J, Reynolds N, Lovat P. NFAT signalling is a novel target of oncogenic BRAF in metastatic melanoma. Br J Cancer 2009;101:1448-55.

8. Kwong LN, Chin L. The brothers RAF. Cell 2010;140:180-2.

9. Davies H, Bignell GR, Cox C, et al. Mutations of the BRAF gene in human cancer. Nature 2002;417:949-54.

10. Ranjbari N, Almasi S, Mohammadi-asl J, Rahim F. BRAF mutations in iranian patients with papillary thyroid carcinoma. Asian Pacif J Cancer Prevent 2013;14:2521-3.

11. Tadmor T, Tiacci E, Falini B, Polliack A. The BRAF-V600E mutation in hematological malignancies: a new player in hairy cell leukemia and Langerhans cell histiocytosis. Leuk Lymphoma 2012;53:2339-40.

12. Ewalt M, Nandula S, Phillips A, et al. Real time PCR based analysis of BRAF V600E mutation in low and intermediate grade lymphomas confirms frequent occurrence in hairy cell leukaemia. Hematol Oncol 2012;30:190-3.

13. Kreitman RJ. Immunoconjugates and new molecular targets in hairy cell leukemia. Hematology Am Soc Hematol Educ Program 2012;2012:660-6. 
14. Tiacci E, Schiavoni G, Forconi F, et al. Simple genetic diagnosis of hairy cell leukemia by sensitive detection of the BRAF-V600E mutation. Blood 2012;119:192-5.

15. Brown NA, Weigelin HC, Bailey N, et al. Requisite analytic and diagnostic performance characteristics for the clinical detection of BRAF V600E in hairy cell leukemia: a comparison of 2 allele-specific PCR Assays. Appl Immunohistochem Mol Morphol 2014 [In press].

16. Verma S, Greaves WO, Ravandi F, et al. Rapid detection and quantitation of BRAF mutations in hairy cell leukemia using a sensitive pyrosequencing assay. Am J Clin Pathol 2012;138:153-6.

17. Rider T, Powell R, Gover R, et al. Molecular detection of BRAF V600E is superior to flow cytometry for disease evaluation in hairy cell leukaemia. Hematol Oncol 2014;32:158-61.

18. Hsieh Y-C, Chang S-T, Chuang S-S, et al. Hairy cell leukemia and variant in Taiwan: report of a variant case and literature review. Int J Clin Exp Patho 2011;4:183.

19. Tiacci E, Schiavoni G, Martelli MP, et al. Constant activation of the RAF-MEK-ERK pathway as a diagnostic and therapeutic target in hairy cell leukemia. Haematologica 2013;98:635-9.

20. Nicolaou F, Teodoridis JM, Park H, et al. CD11c gene expression in hairy cell leukemia is dependent upon activation of the proto-oncogenes ras andjunD. Blood 2003;101:4033-41.

21. Tiacci E, Trifonov V, Schiavoni G, et al. BRAF mutations in hairycell leukemia. N Engl J Med 2011;364:2305-15.

22. Blombery PA, Wong SQ, Hewitt CA, et al. Detection of BRAF mutations in patients with hairy cell leukemia and related lymphoproliferative disorders. Haematologica 2012;97:780-3.

23. Arcaini L, Zibellini S, Boveri E, et al. The BRAF V600E mutation in hairy cell leukemia and other mature B-cell neoplasms. Blood 2012;119:188-91.

24. Xi L, Arons E, Navarro W, et al. Both variant and IGHV4-34-expressing hairy cell leukemia lack the BRAF V600E mutation. Blood 2012;119:3330-2.

25. Tschernitz S, Flossbach L, Bonengel M, et al. Alternative BRAF mutations in BRAF V600E negative hairy cell leukaemias. Br J Haematol 2014;165:529-33.

26. Park JH, Kim E, Chung YR, et al. BRAFV600E mutations occur in the hematopoietic stem cell compartment in hairy cell leukemia. Blood 2013;122:816.

27. Saki N, Abroun S, Hajizamani S, et al. Association of chromosomal translocation and miRNA expression with the pathogenesis of multiple myeloma. Cell 2013;16:2.

28. Saki N, Abroun S, Soleimani M, et al. The roles of miR-146a in the differentiation of Jurkat T-lymphoblasts. Hematology 2014;19:1417.

29. Hosseini Rad SMA, Bavarsad MS, Arefian E, et al. The role of microRNAs in stemness of cancer stem cells. Oncol Rev 2013;7:e8.

30. Chou C-K, Chen R-F, Chou F-F, et al. miR-146b is highly expressed in adult papillary thyroid carcinomas with high risk features including extrathyroidal invasion and the BRAFV600E mutation. Thyroid 2010;20:489-94.

31. Zhou Y-L, Liu C, Dai X-X, et al. Overexpression of miR-221 is associated with aggressive clinicopathologic characteristics and the
BRAF mutation in papillary thyroid carcinomas. Medical Oncol 2012;29:3360-6.

32. Kitagawa Y, Brahmachary M, Tiacci E, et al. A microRNA signature specific for hairy cell leukemia and associated with modulation of the MAPK-JNK pathways. Leukemia 2012;26:2564-7.

33. le Sage C, Nagel R, Egan DA, et al. Regulation of the p27Kip1 tumor suppressor by miR 221 and miR 222 promotes cancer cell proliferation. EMBO J 2007;26:3699-708.

34. Palacios F, Abreu C, Prieto D, et al. Activation of the PI3K/AKT pathway by microRNA-22 results in CLL B-cell proliferation. Leukemia 2014 [Epub ahead of print].

35. Zaidi SK, Dowdy CR, van Wijnen AJ, et al. Altered Runxl subnuclear targeting enhances myeloid cell proliferation and blocks differentiation by activating a miR-24/MKP-7/MAPK network. Cancer Res 2009;69:8249-55.

36. Moussay E, Wang K, Cho J-H, et al. MicroRNA as biomarkers and regulators in B-cell chronic lymphocytic leukemia. Proc Natl Acad Sci 2011;108:6573-8.

37. Holderfield M, Deuker MM, McCormick F, McMahon M. Targeting RAF kinases for cancer therapy: BRAF-mutated melanoma and beyond. Nature Rev Cancer 2014;14:455-67.

38. Samuel J, Macip S, Dyer MJ. Efficacy of vemurafenib in hairy-cell leukemia. N Engl J Med 2014;370:286-8.

39. Santi A, Russo G, Pucciarini A, et al. Targeting The BRAF-MEK-ERK pathway in hairy cell leukemia. Blood 2013;122:3064.

40. Chapman PB, Hauschild A, Robert C, et al. Improved survival with vemurafenib in melanoma with BRAF V600E mutation. $\mathrm{N}$ Engl $\mathrm{J}$ Med 2011;364:2507-16.

41. Dietrich S, Glimm H, Andrulis $\mathrm{M}$, et al. BRAF inhibition in refractory hairy-cell leukemia. N Engl J Med 2012;366:2038-40.

42. Dietrich S, Hüllein J, Hundemer M, et al. Continued response off treatment after BRAF inhibition in refractory hairy cell leukemia. J Clin Oncol 2013;31:e300-e3.

43. Ades F, Metzger-Filho 0. Targeting the cellular signaling: BRAF inhibition and beyond for the treatment of metastatic malignant melanoma. Dermatol Res Pract 2012;2012:259170.

44. King AJ, Patrick DR, Batorsky RS, et al. Demonstration of a genetic therapeutic index for tumors expressing oncogenic BRAF by the kinase inhibitor SB-590885. Cancer Res 2006;66:11100-5.

45. Cantwell-Dorris ER, 0'Leary JJ, Sheils OM. BRAFV600E: implications for carcinogenesis and molecular therapy. Mol Cancer Ther 2011;10:385-94.

46. Pakneshan S, Salajegheh A, Smith RA, Lam AK-Y. Clinicopathological relevance of BRAF mutations in human cancer. Pathol J RCPA 2013;45:346-56.

47. Arcaini L, Zibellini S, Boveri E, et al. The BRAF V600E mutation in hairy cell leukemia and other mature B-cell neoplasms. Blood 2012;119:188-91.

48. Sosman JA, Kim KB, Schuchter L, et al. Survival in BRAF V600 mutant advanced melanoma treated with vemurafenib. $\mathrm{N}$ Engl J Med 2012;366:707-14.

49. Dienstmann R, Tabernero J. BRAF as a target for cancer therapy. Anticancer Agents Med Chem 2011;11:285-95. 